\title{
Escala Argentina de Dificultades Alimentarias en Niños (EADAN): Propiedades psicométricas
}

\author{
Argentine Scale of Feeding Difficulties in Children (EADAN): \\ Psychometric properties
}

\author{
Maria Paulina Hauser * 1, Ruth Alejandra Taborda ${ }^{1}$, \\ Alicia Oiberman ${ }^{2}$, Maria Ramsay ${ }^{3}$ \\ 1 - Facultad de Psicología, Universidad Nacional de San Luis. \\ 2 - CIIPME - CONICET. Facultad de Psicología, Universidad de Buenos Aires. \\ 3 - Departamento de Psicología, Hospital de Niños de Montreal. Departamento de Pediatría, \\ Universidad McGill, Montreal, Quebec.
}

Recibido: 31/01/2019 Revisado: 03/03/2019 Aceptado: 06/03/2019

\section{Resumen}

El objetivo de esta investigación fue adaptar y validar en Argentina un cuestionario para padres que detecta dificultades en la alimentación, las cuales son muy comunes entre los niños pequeños. Se analizaron las propiedades psicométricas de la Escala Argentina de Dificultades Alimentarias en Niños (EADAN) en una muestra argentina de 263 niños de entre los 6 meses y los 6 años 11 meses de edad. Existen diferencias significativas entre la media por ítem y la media del puntaje total en los grupos con dificultades y sin dificultades en la alimentación. La confiabilidad de la escala es buena, con un valor $\alpha=.79$. Se realizó un análisis factorial exploratorio que arrojó dos factores que representan el $49.4 \%$ de la varianza. Se compararon los puntajes obtenidos por niños prematuros y nacidos a término. Se concluye acerca de la validez del instrumento para la detección de dificultades en la alimentación en niños argentinos.

Palabras clave: problemas de alimentación, niños, cuestionario, Argentina, niños prematuros, niños nacidos a término

\section{Summary}

The objective of this study was to adapt and validate in Argentina a questionnaire for parents that detects feeding difficulties, which are very common among young children. Psychometric properties of the Argentine Scale of Feeding Difficulties in Children (EADAN) were analyzed in an Argentinian sample of 263 children, aged 6 months to 6 years 11 months. There are significant differences between the item mean and the total score mean between the groups with feeding difficulties and without feeding difficulties. The reliability of the scale is good, with an $\alpha=.79$. An exploratory factor analysis showed two factors that represent $49.4 \%$ of the variance. Scores obtained by premature and full-term infants were compared. It is concluded that this instrument is valid for the detection of feeding difficulties in Argentine children.

Key words: feeding problems, children, questionnaire, Argentina, premature, full-term

*Correspondencia a: María Paulina Hauser. Facultad de Psicología, Universidad Nacional de San Luis. CIIPME - CONICET. Tte. Gral. Juan Domingo Perón 2158, C1040AAH CABA. Buenos Aires, Argentina. Tel: +54 (011) 4953-1477/3541. E-mail: mphauser@unsl.edu.ar

Nota de autor: Se agradece a las instituciones en las que se realizó la investigación y al Consejo Nacional de Investigaciones Científicas y Técnicas (CONICET) por el financiamiento de la misma mediante una Beca Postdoctoral.

Cómo citar este artículo: Hauser, M. P., Taborda, R. H., Oiberman, A., \& Ramsay, M. (2019). Escala Argentina de Dificultades Alimentarias en Niños (EADAN): Propiedades psicométricas. Revista Evaluar, 19(2), 1-14. Recuperado de htts://revistas.unc.edu.ar/index.php/revaluar 


\section{Introducción}

Los problemas de alimentación son muy comunes entre los niños pequeños, tienen un impacto negativo en su desarrollo y están asociados a un mayor estrés durante el momento de la alimentación, pobre crecimiento y retraso en el desarrollo cognitivo (van Dijk, Timmerman, Martel, \& Ramsay, 2011). Las dificultades en la alimentación y la ganancia de peso en la infancia generan intensas preocupaciones en los padres, por lo que son motivos frecuentes de consulta en los servicios pediátricos. Entre los niños con un desarrollo típico, se estima que las dificultades de alimentación se presentan en el $25 \%$ al $45 \%$ de los niños con desarrollo típico, mientras que se dan en un $30 \%$ al $80 \%$ en niños con trastornos del desarro11 (Arvedson, como se citó en Barton, Bickell, \& Fucile, 2017).

Las dificultades en torno a la alimentación que pueden presentar los niños abarcan desde la ingesta en cantidades limitadas o sólo de cierto tipo de alimentos hasta la imposibilidad de comer por sí mismos y de manejar la comida o los utensilios apropiadamente. Si bien en muchos casos estas complicaciones pasan desapercibidas, pueden afectar de manera significativa la salud física y psicosocial de los niños y sus padres (Adamson, Morawska, \& Sanders, 2013). En un estudio realizado en Singapur se entrevistó a 407 cuidadores, quienes informaron que el comportamiento quisquilloso al comer y las dificultades aparecieron alrededor del primer año de vida de los niños. La mayor tendencia a presentar dificultades en la alimentación se observó en niños de entre 3 y 10 años, en niños con padres profesionales y en aquellos que tenían antecedentes familiares de dificultades en la alimentación. El comportamiento quisquilloso al comer y las dificultades en la alimentación se asociaron significativamente con el estrés de los cuidadores durante las comidas y el impacto negativo en las relaciones familiares (Goh \& Jacob, 2012).

Los problemas de alimentación en la infancia se explican desde un modelo biopsicosocial, lo que supone que son el resultado del interjuego entre factores fisiológicos y psicosociales (Johnson \& Harris, 2004; Rommel, De Meyer, Feenstra, \& Veereman-Wauters, 2003). Los factores fisiológicos que pueden contribuir al desarrollo de problemas en la alimentación incluyen la prematurez, el poco apetito y las dificultades en la sensomotricidad oral, mientras que los factores psicosociales incluyen el temperamento del niño y las dificultades en la relación madre-hijo (van Dijk et al., 2011). La relación que se establece entre la madre y el niño en torno a la alimentación está influenciada tanto por factores fisiológicos como interactivos (Ramsay, 2013).

El factor conductual es señalado como central en la aparición de dificultades en la alimentación de los niños junto con ciertos factores ambientales y conductuales de los padres que parecen predecir la mayor o menor aparición de dificultades. Entre las conductas parentales que aumentarían las dificultades se señalan: proporcionar comidas alternativas, amenazar y presionar al niño para comer, el castigo físico y las reprimendas (Adamson et al., 2013). Las estrategias o conductas utilizadas usualmente por los padres para facilitar o limitar la ingesta de ciertos alimentos se denominan prácticas de alimentación, dentro de las cuales las más estudiadas son la restricción y la presión para comer. Por lo general, estas prácticas responden a características o conductas específicas del niño, como por ejemplo ser quisquilloso para comer (Blisseth, 2011). El estilo adoptado para alimentar a los niños parece estar influido en cierta medida por el nivel educativo materno. En una investigación realizada en el Reino Unido, se obtuvo que las madres con mayor nivel de estudios suelen tener más control sobre la comida y 
recurren significativamente menos a la alimentación emocional, en la cual los alimentos se ofrecen para calmar al niño en lugar de saciar su apetito. Las estrategias de alimentación emocional y de alimentación instrumental, en la que la comida es ofrecida como recompensa o retirada a modo de castigo, suelen ser perjudiciales para el manejo adecuado del peso de los niños (Saxton, Carnell, van Jaarsveld, \& Wardle, 2009).

En una investigación reciente realizada en Dinamarca, se investigaron los factores perinatales de riesgo asociados a la aparición de desórdenes en la alimentación en niños de 0 a 3 años. Los investigadores hallaron que los niños nacidos antes de las 28 semanas de edad gestacional (EG), los pequeños para su edad gestacional y los infantes con malformaciones tenían el mayor riesgo de presentar este tipo de desórdenes. Además, la pertenencia al sexo femenino, el hábito materno de fumar durante el embarazo y el estatus de inmigrante y de primer hijo parecerían estar asociados con el incremento del riesgo de desórdenes en la alimentación en la población estudiada. En cuanto a la prevalencia de los desórdenes en la alimentación, se encontró que la mayoría de los niños eran diagnosticados durante los primeros 12 meses de vida, en particular en los primeros 4 meses. Los prematuros fueron quienes tenían más probabilidades de ser diagnosticados, probablemente debido a problemas neurológicos, como consecuencia de la alimentación mediante sonda naso u orogástrica y de las características de la relación temprana madre-hijo (Hvelplund, Hansen, Vinkel-Koch, Andersson, \& Skovgaard, 2016). En una investigación reciente conducida en niños prematuros (Van Dijk, Bruinsma, \& Hauser, 2016), hallaron que a medida que los padres informaban mayores problemas en la alimentación, los niños observados rechazaban en mayor medida los alimentos y comían de manera menos eficiente e independiente. Los niños prematuros de este estudio presenta- ron puntajes que indicaban ausencia de problemas en la alimentación o problemas leves.

Dado que la comunidad científica no posee aún una definición aceptada de manera universal respecto de las dificultades en la alimentación, los investigadores han utilizado diversos términos para referirse a los comportamientos que indicarían dichos problemas (Van Dijk et al., 2011). Se señalan como indicadores de dificultades en la alimentación en niños: el rechazo de la comida (Lindberg, Bohlin, \& Hagekull, 1991), la irregularidad al comer (McDermott et al., 2008), la desobediencia durante la alimentación (Sanders, Patel, Le Grice, \& Shepherd, 1993) y la negatividad a la hora de comer (Johnson \& Harris, 2004). Empujar los alimentos fuera de la boca, tener arcadas, guardar comida en la boca sin tragarla y llorar también han sido descritos como determinantes para considerar si un niño tiene problemas de alimentación (Chung et al., como se citó en Fernández-Gallardo, Rojas-Contreras, \& Vargas-Keith, 2017). Si bien los comportamientos problemáticos en torno a la alimentación (por ejemplo, girar la cabeza, arquear el cuerpo o llorar) tienden a ser reacciones a estímulos internos en un primer momento (falta de hambre, falta de capacidad de succión), también pueden llegar a ser condicionados por la asociación a las señales externas y sociales (por ejemplo, engaños de parte de los padres). La detección temprana puede mejorar los comportamientos durante la alimentación y las interacciones a la hora de la comida, que a su vez ayudan a promover la independencia y otras habilidades en el niño (Ramsay, 2013).

Debido a la prevalencia de los problemas de alimentación y sus consecuencias negativas en el desarrollo de los niños pequeños, resulta de gran importancia que los profesionales que trabajan en primera infancia cuenten con instrumentos que permitan la rápida detección de dificultades en la alimentación en niños. Con el propósito de res- 
ponder a esta vacancia, Ramsay, Martel, Porporino y Zygmuntowicz (2011) diseñaron en Canadá la escala Montreal Children's Hospital Feeding Scale (MCH-FS). Este es un instrumento de fácil administración, cuyo propósito es detectar rápidamente dificultades en la alimentación en niños pequeños en una consulta breve.

La MCH-FS está compuesta por 14 ítems que fueron construidos por psicólogos expertos en el trabajo con niños con problemas en la alimentación. Estos ítems indagan en diferentes aspectos de la alimentación: lo oromotor, lo sensoriomotor, el apetito, las preocupaciones maternas sobre la alimentación, los comportamientos en el momento de la alimentación, las estrategias utilizadas por la madre y las reacciones familiares ante la alimentación del niño. Se le solicita a la persona encargada de alimentar al niño que puntúe los 14 ítems en una escala Likert de siete puntos, con puntos de atracción en cada extremo. El cuidador puntúa cada ítem de acuerdo a la frecuencia o el nivel de dificultad en un comportamiento particular o según el nivel de preocupación parental. Se obtiene un puntaje total a partir de la suma de todos los ítems. Ese puntaje bruto luego se convierte en un puntaje T que indicaría dificultades leves, moderadas o severas en la alimentación. A mayor puntaje, mayor la gravedad de las dificultades.

El instrumento fue sometido a estudios de validez y confiabilidad. A fin de establecer la validez de constructo, se administró el cuestionario a 198 madres de niños que asistían al consultorio pediátrico (muestra normativa) y a 174 madres de niños derivados a una clínica especializada en desórdenes de la alimentación (muestra clínica). Se obtuvo una excelente validez de constructo, ya que al comparar las puntuaciones medias de las muestras normativas y clínicas se observaron claras diferencias $(\mathrm{M}=32.65, \mathrm{DE}=12.73$ contra $\mathrm{M}$ $=60.48, \mathrm{DE}=13.04$ respectivamente; $p<.01)$.
La confiabilidad test-retest fue buena para ambos grupos (normativo $r=.84$ y clínico $r=.92$ ). El análisis de factor realizado mediante el análisis de componentes principales evidenció que un solo factor representaba al $48 \%$ de la varianza, lo que sugiere que el puntaje total obtenido en la escala puede ser utilizado como una medida de los problemas de alimentación. Los resultados evidencian que los niños en el grupo clínico presentan los mismos problemas en la alimentación que los niños del grupo control, pero en mayor frecuencia o de manera más severa (Ramsay et al., 2011).

Este instrumento MCH-FS también fue traducido y estandarizado en los Países Bajos y en Tailandia. La versión holandesa del instrumento, denominada Screeningslijst Eetgedrag Peuters (SEP; Van Dijk et al., 2011), fue sometida a estudios de validez y confiabilidad en una muestra normativa de 1448 niños entre los 6 meses y los 4 años que residían en la provincia de Groninga de los Países Bajos. Los resultados evidencian una buena confiabilidad (alfa de Cronbach $\alpha=.84)$. En contraste con la investigación de los creadores de la escala, a partir del análisis factorial exploratorio mediante componentes principales se obtuvieron dos factores que se corresponderían significativamente con 12 de los 14 ítems de la MCH-FS y que representan una varianza del $43 \%$. La correlación entre los dos factores es alta, de .69. Los autores consideran que el primer factor reúne los comportamientos negativos $d u$ rante las comidas mientras que el segundo factor incluye aquellos ítems que indicarían causas y consecuencias negativas de las dificultades en la alimentación. A partir del análisis de las propiedades psicométricas, se puede concluir que la SEP es un instrumento válido para la detección de problemas en la alimentación en niños.

La versión tailandesa de la MCH-FS (Benjasuwantep, Rattanamongkolgul, \& Ramsay, 2015) fue desarrollada a partir de una muestra de 200 
niños de entre los 12 y los 48 meses. La confiabilidad fue alta, con un alfa de Cronbach de .83. El análisis factorial se realizó utilizando el análisis de componentes principales, lo que resultó en tres factores que dan cuenta del $52.3 \%$ de la varianza, con una correlación moderada entre los tres factores. Los primeros dos factores están relacionados con causas de comportamientos negativos durante las comidas y preocupaciones y reacciones parentales, el tercer factor contempla efectos de las situaciones de alimentación negativas, con una correlación moderada entre los tres factores. Es interesante señalar que los autores debieron realizar una modificación en el ítem 5 introduciendo la opción Nunca finaliza la comida.

A partir de la revisión de los instrumentos de los que se dispone en nuestro medio, se tomó conocimiento de la ausencia de pruebas estandarizadas en nuestra población para la detección de las dificultades en la alimentación en la primera infancia. Es por esto que el objetivo de esta investigación fue adaptar y validar en Argentina el cuestionario para padres MCH-FS (Ramsay et al., 2011). El presente trabajo se propone analizar las propiedades psicométricas de la versión local denominada Escala Argentina de Dificultades Alimentarias en Niños (EADAN) en una muestra obtenida de la población argentina.

\section{Metodología \\ Participantes}

Para la selección de participantes se instrumentó un proceso bietápico. En la primera etapa se escogió en la ciudad de San Luis, provincia de San Luis, una clínica privada y la Maternidad Provincial Dra. Teresita Baigorria. En la segunda etapa se seleccionó una Maternidad pública en la ciudad de Avellaneda, provincia de Buenos Aires. Dichas instituciones fueron escogidas por ser cen- tros de referencia en las zonas en las que se llevó a cabo la investigación.

La muestra está formada por 263 niños de entre los 6 meses y los 6 años y 11 meses de edad. Se incluyeron 177 niños nacidos a término y 86 niños prematuros. Los niños prematuros se clasificaron según su edad gestacional (EG) al nacer en prematuros extremos (menos de 28 semanas de EG al nacer), muy prematuros (entre 28 y 31 semanas de EG) y moderados (entre 32 y 36 semanas de EG). Se excluyeron niños que habían recibido previamente un diagnóstico de desorden en la alimentación o se encontraban en tratamiento debido a problemas de alimentación. Se solicitó el consentimiento informado a los padres de todos los niños involucrados.

\section{Instrumentos}

Se utilizó la Escala Argentina de Dificultades Alimentarias en Niños (EADAN), versión argentina del cuestionario MCH-FS (Ramsay et al., 2011). Para validar el instrumento, primero fue necesario adaptarlo culturalmente a la población local en la que se pretendía utilizar y luego volver a medir sus propiedades psicométricas. El MCH-FS fue traducido con el permiso de la autora creadora del instrumento, quien además es una de las autoras del presente artículo. La versión en inglés del MCH-FS fue traducida al español por un traductor bilingüe cuya lengua materna es el español. Luego fue retraducida al inglés por dos traductores bilingües, uno cuya lengua materna es el inglés y en el otro caso, el español. Estas versiones fueron comparadas entre sí para evaluar la concordancia con la versión original. Fue necesario introducir ciertas modificaciones en la versión en español a fin de utilizar palabras que fueran más frecuentes en el uso cotidiano y, por ende, más fáciles de comprender. Estos cambios fueron 
consultados con la autora original, quien consideró que eran pertinentes si contribuían a mejorar la comprensión en el idioma español. La versión final en español fue enviada a tres expertos en la temática a fin de estudiar la pertinencia de los ítems indagados en nuestra población local. Según los expertos, todos los ítems reflejan nuestra cultura, así como las dificultades en torno a la alimentación, y se encuentran redactados de forma tal que pueden ser comprendidos por los padres.

Junto con el cuestionario EADAN, se les entregó a los padres de los niños un cuestionario sociodemográfico, elaborado por los autores de este artículo, que permitió obtener información sobre la edad del padre o madre, nivel educativo, situación laboral y lugar de residencia. También permitió recabar información sobre la edad del niño, la fecha de nacimiento, la edad gestacional al nacer, el peso y la talla de nacimiento, si estuvo internado en la UCIN (tiempo de internación, motivo, asistencia respiratoria utilizada, alimentación por sonda), peso y talla actual del niño, si recibe algún tipo de suplemento nutricional, si padece de alguna enfermedad crónica (medicación), si come junto con la familia o por separado, el nivel de desarrollo alcanzado y antecedentes familiares de trastornos de la alimentación.

\section{Procedimiento}

Los cuestionarios fueron entregados a los padres de los niños que asistían a instituciones de salud en las ciudades de San Luis y Avellaneda en el periodo comprendido entre junio de 2017 y noviembre de 2018. Las tres instituciones poseen características diferentes. Una es una institución privada ubicada en la zona céntrica de la ciudad de San Luis, a la que asisten en su gran mayoría personas que poseen obra social y tienen trabajos estables. Otra es una institución pública de referencia a nivel provincial, por lo que asisten personas de diversos lugares del interior que, en general, no poseen obra social y se encuentran en una situación socioeconómica difícil. Esta institución recibe además a niños nacidos prematuros o de alto riesgo en su Consultorio de Seguimiento para Niños de Alto Riesgo. La tercera institución se encuentra ubicada en el primer cordón del conurbano bonaerense y asiste a una población que no posee, en su amplia mayoría, obra social y se encuentra en situación de vulnerabilidad social, económica y cultural. Este hospital también posee un Consultorio de Seguimiento al que asisten niños de Alto Riesgo.

Los padres podían responder el cuestionario en el tiempo de espera para ingresar a la consulta pediátrica, o bien luego de la misma. Si bien se trata de un cuestionario autoadministrado, en todos los casos el primer investigador asistió a los padres durante la respuesta del mismo a fin de aclarar si surgía alguna duda y asegurarse de que los cuestionarios fueran completados en su totalidad. Previo a la respuesta del cuestionario se les informó a los sujetos acerca del objetivo de la investigación, así como que su participación era voluntaria y anónima, y se les solicitó la firma del consentimiento informado. Todos los cuestionarios fueron ingresados de forma manual en el paquete estadístico SPSS versión 21 (IBM, 2012). En los ítems 1, 3, 4, 8, 10, 12 y 13 el puntaje se ingresó de manera invertida tal como indica la versión original para la puntuación; en el caso de estos ítems se utilizaron para el análisis los valores invertidos.

\section{Análisis estadístico}

El análisis estadístico de los ítems individuales y del puntaje total de la escala se realizó en SPSS versión 21 (IBM, 2012). El análisis fac- 
torial exploratorio se realizó en el programa Factor (Lorenzo-Seva \& Ferrando, 2013). La adecuación del análisis factorial se calculó a través de la medida KMO de Kaiser y de esfericidad de Bartlett. El método de estimación de factores utilizado fue el de mínimos cuadrados no ponderados. La selección del número de factores se realizó mediante el procedimiento de análisis paralelo (AP; Horn, 1965). La rotación factorial fue oblicua y se utilizó el criterio promin (Lorenzo-Seva, 1999). La confiabilidad se determinó mediante el coeficiente alfa de Cronbach. La validez de criterio se estableció mediante la comparación de la media del puntaje total y la del puntaje por ítem entre el grupo de niños que presentaron dificultades en la alimentación y en el grupo que no presentó dificultades según el cuestionario.

\section{Resultados}

Descripción de los items individuales y el puntaje total

Doscientos treinta niños de la muestra no presentaron dificultades en la alimentación, mientras que dentro del grupo de los que sí presentaron dificultades se discriminó a dieciocho niños con dificultades leves, ocho con dificultades moderadas y siete con dificultades severas.

En la muestra total, el puntaje medio para los 14 ítems va desde 1.45 a 3.10. Estos valores son consistentes con los valores promedio obtenidos en la muestra normativa de Canadá, así como en los estudios de validación en los Países Bajos y Tailandia. El análisis descriptivo también puso en evidencia que los puntajes por ítem, así como el puntaje total, se distribuían claramente de forma positiva (ver Tabla 1).

Se hallaron diferencias significativas al comparar la media de los puntajes por ítem y del puntaje total en el grupo de niños sin dificulta-
Tabla 1

Media de los ítems individuales y del puntaje total de la EADAN para la muestra total $(\mathrm{N}=263)$.

\begin{tabular}{lccc}
\hline $\begin{array}{c}\text { Ítems } \\
\text { EADAN }\end{array}$ & Media & Desv. típ. & Asimetría \\
\hline Ítem 1 & 2.67 & 1.62 & 0.79 \\
Ítem 2 & 2.39 & 1.91 & 1.04 \\
Ítem 3 & 2.23 & 1.57 & 1.14 \\
Ítem 4 & 3.10 & 2.53 & 0.64 \\
Ítem 5 & 2.66 & 1.29 & 1.14 \\
Ítem 6 & 2.59 & 1.70 & 0.72 \\
Ítem 7 & 1.87 & 1.60 & 2.01 \\
Ítem 8 & 1.65 & 1.46 & 2.44 \\
Ítem 9 & 2.62 & 2.10 & 0.96 \\
Ítem 10 & 1.85 & 1.69 & 2.05 \\
Ítem 11 & 1.45 & 1.10 & 2.68 \\
Ítem 12 & 1.58 & 1.26 & 2.61 \\
Ítem 13 & 1.60 & 1.37 & 2.42 \\
Ítem 14 & 1.71 & 1.47 & 2.13 \\
Puntaje total & 30.04 & 12.30 & 1.16 \\
\hline
\end{tabular}

des y en el de aquellos con dificultades. Se observa que el grupo de niños con dificultades obtuvo puntajes significativamente superiores en todos los ítems de la escala. El ítem 5 es el único en el que la diferencia entre los dos grupos no resulta tan significativa. La media del puntaje total en el grupo de niños con dificultades duplica el valor del grupo de niños sin dificultades (ver Tabla 2).

Respecto a los aspectos sociodemográficos, en los resultados se puede observar que las madres del grupo de niños que presentaron dificultades poseen en su mayoría estudios secundarios completos (48\%), en menor medida estudios terciarios o universitarios completos $(21 \%)$ y estudios secundarios incompletos (18\%). La gran mayoría de estas madres eran amas de casa (46\%) mientras que el resto tenía trabajos inestables $(21 \%)$ o un trabajo estable (33\%). No se obtuvie- 
Tabla 2

Media de los ítems individuales y del puntaje total de la EADAN para los grupos sin dificultades y con dificultades.

\begin{tabular}{|c|c|c|c|c|}
\hline \multirow{2}{*}{$\begin{array}{l}\text { Ítems } \\
\text { EADAN }\end{array}$} & \multicolumn{2}{|c|}{$\begin{array}{c}\text { Sin Dificultades } \\
(n=230)\end{array}$} & \multicolumn{2}{|c|}{$\begin{array}{l}\text { Con Dificultades } \\
(\mathbf{n}=\mathbf{3 3})\end{array}$} \\
\hline & Media & DE & Media & DE \\
\hline Ítem 1 & 2.34 & 1.36 & 4.94 & 1.49 \\
\hline Ítem 2 & 2.12 & 1.73 & 4.27 & 2.08 \\
\hline Ítem 3 & 1.94 & 1.31 & 4.21 & 1.79 \\
\hline Ítem 4 & 2.71 & 2.37 & 5.82 & 1.89 \\
\hline Ítem 5 & 2.62 & 1.21 & 2.94 & 1.73 \\
\hline Ítem 6 & 2.37 & 1.54 & 4.15 & 1.97 \\
\hline Ítem 7 & 1.68 & 1.41 & 3.21 & 2.13 \\
\hline Ítem 8 & 1.50 & 1.21 & 2.73 & 2.40 \\
\hline Ítem 9 & 2.24 & 1.84 & 5.30 & 1.79 \\
\hline Ítem 10 & 1.45 & 1.13 & 4.64 & 2.21 \\
\hline Ítem 11 & 1.35 & 0.96 & 2.15 & 1.66 \\
\hline Ítem 12 & 1.45 & 1.08 & 2.52 & 1.88 \\
\hline Ítem 13 & 1.30 & 0.92 & 3.64 & 2.08 \\
\hline Ítem 14 & 1.50 & 1.24 & 3.15 & 2.01 \\
\hline $\begin{array}{l}\text { Puntaje } \\
\text { total }\end{array}$ & 26.53 & 8.06 & 54.48 & 8.28 \\
\hline
\end{tabular}

ron diferencias significativas en relación con el sexo del niño, ya que un $58 \%$ eran varones y un $42 \%$ eran mujeres.

En cuanto a los antecedentes neonatales de los niños, se encontró que la gran mayoría nació a término (23 niños, 70\%), en segundo lugar se encontraron los prematuros moderados (seis niños, $18 \%$ ) y en igual cantidad los muy prematuros y los extremos (dos niños respectivamente, 6\%). El peso al nacer tampoco parecería influir demasiado en este grupo, ya que el $70 \%$ tuvo un peso adecuado, mientras que el $12 \%$ tuvo muy bajo peso, y el $9 \%$ de los niños con dificultades nacieron con bajo peso y extremo bajo peso respectivamente. La mayoría de estos infantes no permanecieron internados en la UCIN al nacer (20 niños, 60\%), no fueron alimentados mediante sonda orogástrica (24 niños, 73\%), ni requirieron asistencia respiratoria (24 niños, 73\%). Se observa un leve predominio de niños que asisten a la guardería o a la escuela (17 niños, 52\%). La mayoría de los niños come junto a la familia (26 niños, 79\%) y no poseen antecedentes familiares de trastornos en la alimentación (24 niños, 73\%).

Al dividir la muestra en grupos de edad se puede notar que el puntaje medio obtenido en la escala aumenta considerablemente desde los 6 meses hasta los 4 años. De igual manera, también se incrementa la desviación estándar, lo que indica que a medida que los niños crecen, las diferencias inter-individuales son mayores. Si bien la media del puntaje total alcanza su pico a los 4 años, luego desciende levemente entre los 4 años 1 mes y los 5 años para volver incrementarse hasta los 6 años y 11 meses.

Por otra parte, se puede advertir que en el grupo de niños que presentaron dificultades en la alimentación, la mayor concentración se obtuvo en el rango de 6 meses a 1 año (ocho niños), entre los 2 años y 1 mes - 3 años (seis niños) y entre los 4 años y 1 mes - 5 años (seis niños). Esto podría indicar que en la muestra estudiada la mayoría de los infantes presentaron dificultades en el periodo de introducción de los alimentos semisólidos. Sin embargo, al considerar la media del puntaje total obtenido en la EADAN, se observa que los valores más elevados se encuentran en el rango 3 años y 1 mes - 4 años, seguido por el valor medio del rango 4 años y 1 mes - 5 años (ver Tabla 3).

\section{Diferencias entre los niños nacidos prematuros y los nacidos a término}

Se realizó una comparación de los puntajes obtenidos por los niños nacidos prematuros y los niños nacidos a término. Se encontró que el 12\% 
Tabla 3

Media del puntaje total según grupos de edad en la muestra total y en los niños con dificultades en la alimentación.

\begin{tabular}{|c|c|c|c|}
\hline $\begin{array}{l}\text { Grupos } \\
\text { (años y meses) }\end{array}$ & Media & Desv. típ. & $N=263$ \\
\hline 6 meses a 1 año & 26.77 & 11.80 & 74 \\
\hline $\begin{array}{l}1 \text { año y } 1 \text { mes a } \\
2 \text { años }\end{array}$ & 27.36 & 8.67 & 45 \\
\hline $\begin{array}{l}2 \text { años y } 1 \text { mes a } \\
3 \text { años }\end{array}$ & 32.45 & 12.21 & 47 \\
\hline $\begin{array}{l}3 \text { años y } 1 \text { mes a } \\
4 \text { años }\end{array}$ & 35.18 & 14.74 & 28 \\
\hline $\begin{array}{l}4 \text { años y } 1 \text { mes a } \\
5 \text { años }\end{array}$ & 30.74 & 15.42 & 27 \\
\hline $\begin{array}{l}5 \text { años y } 1 \text { mes a } \\
6 \text { años }\end{array}$ & 31.50 & 13.50 & 16 \\
\hline $\begin{array}{l}6 \text { años y } 1 \text { mes } \\
\text { a } 6 \text { años y } 11 \\
\text { meses }\end{array}$ & 33.39 & 9.28 & 23 \\
\hline
\end{tabular}

\begin{tabular}{|c|c|c|c|}
\hline $\begin{array}{l}\text { Niños con } \\
\text { dificultades en } \\
\text { la alimentación }\end{array}$ & Media & Desv. típ. & $\mathbf{n}=\mathbf{3 3}$ \\
\hline 6 meses a 1 año & 53.38 & 6.61 & 8 \\
\hline $\begin{array}{l}1 \text { año y } 1 \text { mes a } \\
2 \text { años }\end{array}$ & 48 & 1.41 & 2 \\
\hline $\begin{array}{l}2 \text { años y } 1 \text { mes a } \\
3 \text { años }\end{array}$ & 55 & 9.63 & 6 \\
\hline $\begin{array}{l}3 \text { años y } 1 \text { mes a } \\
4 \text { años }\end{array}$ & 59.80 & 13.88 & 5 \\
\hline $\begin{array}{l}4 \text { años y } 1 \text { mes a } \\
5 \text { años }\end{array}$ & 55.67 & 6.91 & 6 \\
\hline $\begin{array}{l}5 \text { años y } 1 \text { mes a } \\
6 \text { años }\end{array}$ & 48.75 & 1.50 & 4 \\
\hline $\begin{array}{l}6 \text { años y } 1 \text { mes } \\
\text { a } 6 \text { años y } 11 \\
\text { meses }\end{array}$ & 50.50 & 2.12 & 2 \\
\hline
\end{tabular}

de los niños que nacieron prematuros tuvieron dificultades en la alimentación según el instrumento, mientras que estas dificultades aparecieron en el $13 \%$ de los niños nacidos a término.
En el grupo de prematuros extremos el rango de EG al nacer abarcó entre 25 y 27 semanas de gestación, con un valor medio de 26.25 semanas. La EG media en los niños nacidos muy prematuros fue de 29.76 con un rango de edad de entre 28 y 31 semanas. En los prematuros moderados la media fue de 34.42 con un rango de edad entre 32 y 36 semanas. Finalmente, en el grupo de nacidos a término la EG media fue de 39.10 semanas con un rango de edad entre 37 y 42 semanas de gestación.

Se observa que el grupo de los prematuros extremos obtuvo el puntaje medio total más alto de la muestra con un valor de 32 puntos. También se puede notar que los prematuros extremos tuvieron el puntaje medio por ítem más alto en siete de las variables estudiadas, particularmente en aquellas que se refieren a conductas como negarse a comer, irregularidad al comer, mal comportamiento durante las comidas, guardar la comida en la boca sin tragarla; así como también en los ítems que indicarían dificultades en las habilidades oromotoras y pobre crecimiento. En cambio, se observa que en este grupo la alimentación no afectaría las relaciones con los padres y la familia en su totalidad, probablemente debido a la atención especial que recibieron durante la internación y en el seguimiento.

Los niños considerados como muy prematuros obtuvieron el puntaje medio por ítem más alto en cuatro de las variables indagadas, en especial en aquellas que remiten a tener arcadas, escupir o vomitar con cierto tipo de comidas y la necesidad de entretenerlo o perseguirlo para que coma, así como la necesidad de forzarlo a hacerlo. Finalmente, el grupo de prematuros moderados y los nacidos a término obtuvieron los puntajes medios más altos sólo en dos de los ítems del cuestionario (ver Tabla 4). 
Tabla 4

Comparación entre nacidos prematuros y nacidos a término respecto de los puntajes medios por ítem y total de la EADAN.

\begin{tabular}{|c|c|c|c|c|c|c|c|c|}
\hline \multirow[t]{2}{*}{ Ítems } & \multicolumn{2}{|c|}{$\begin{array}{l}\text { Prematuros extremos } \\
\qquad \mathbf{n}=\mathbf{8}\end{array}$} & \multicolumn{2}{|c|}{$\begin{array}{l}\text { Muy Prematuros } \\
\qquad \mathbf{n}=\mathbf{2 1}\end{array}$} & \multicolumn{2}{|c|}{$\begin{array}{l}\text { Prematuros } \\
\text { Moderados } \\
\mathbf{n}=\mathbf{5 7}\end{array}$} & \multicolumn{2}{|c|}{$\begin{array}{l}\text { Nacidos a término } \\
\qquad \mathrm{n}=177\end{array}$} \\
\hline & Media & Desv. típ. & Media & Desv. típ. & Media & Desv. típ. & Media & Desv. típ. \\
\hline Ítem 1 & 2.63 & 2.387 & 2.62 & 1.465 & 2.30 & 1.476 & 2.80 & 1.649 \\
\hline Ítem 2 & 2.75 & 1.909 & 2.14 & 1.957 & 2.33 & 2.099 & 2.42 & 1.867 \\
\hline Ítem 3 & 1.88 & 1.246 & 1.86 & 1.153 & 2.39 & 1.770 & 2.24 & 1.559 \\
\hline Ítem 4 & 4.25 & 3.012 & 2.76 & 2.587 & 2.53 & 2.315 & 3.27 & 2.562 \\
\hline Ítem 5 & 3.25 & 1.165 & 2.86 & 1.621 & 2.65 & 1.395 & 2.62 & 1.220 \\
\hline Ítem 6 & 2.88 & 2.167 & 2.67 & 1.653 & 2.16 & 1.590 & 2.71 & 1.720 \\
\hline Ítem 7 & 2.13 & 2.232 & 2.57 & 1.777 & 1.86 & 1.586 & 1.78 & 1.546 \\
\hline Ítem 8 & 2.13 & 2.100 & 1.76 & 1.609 & 1.70 & 1.488 & 1.60 & 1.423 \\
\hline Ítem 9 & 1.88 & 1.642 & 2.67 & 2.033 & 2.53 & 2.292 & 2.68 & 2.070 \\
\hline Ítem 10 & 1.38 & 1.061 & 2.14 & 2.242 & 1.82 & 1.794 & 1.84 & 1.609 \\
\hline Ítem 11 & 1.75 & 1.753 & 1.14 & .359 & 1.51 & 1.325 & 1.46 & 1.050 \\
\hline Ítem 12 & 2.63 & 1.923 & 1.81 & 1.209 & 1.68 & 1.572 & 1.47 & 1.093 \\
\hline Ítem 13 & 1.50 & 1.414 & 1.24 & .768 & 1.67 & 1.574 & 1.62 & 1.360 \\
\hline Ítem 14 & 1.00 & .000 & 1.86 & 1.740 & 1.49 & 1.151 & 1.79 & 1.551 \\
\hline Puntaje total & 32.00 & 12.189 & 30.10 & 11.717 & 28.49 & 13.505 & 30.45 & 12.026 \\
\hline
\end{tabular}

Propiedades psicométricas de la escala

Se analizó la confiabilidad de la escala mediante el estadístico alfa de Cronbach, a partir de lo cual se obtuvo que la misma es buena con un valor $\alpha=.79$. Se realizó un análisis factorial exploratorio en el programa Factor (Lorenzo-Seva \& Ferrando, 2013). El estadístico KMO arrojó un valor de .85 , por lo que se considera satisfactorio, y en la prueba de Bartlett la significación fue de $p<.001$. A partir de estos estadísticos se pudo comprobar la adecuación de los datos al análisis factorial, por lo que resulta apropiado realizarlo. El método de extracción de factores utilizado fue el de mínimos cuadrados no ponderados. Se seleccionó el número de factores mediante el proce- dimiento de análisis paralelo (AP), que arrojó dos factores que representan el $49.4 \%$ de la varianza. Se retuvieron aquellos ítems cuya saturación fue superior a .30 (ver Tabla 5). La correlación entre los dos factores es alta, de .76.

La estructura factorial subyacente permite establecer que el primer factor agrupa los ítems referidos a causas y consecuencias de las dificultades en la alimentación; entre ellas se puede mencionar el poco apetito, la negativa a comer, las habilidades oromotoras y orosensoriales deficientes y el pobre crecimiento del niño. El segundo factor abarca los ítems que se refieren a preocupaciones parentales y estrategias utilizadas frente a las dificultades en la alimentación, tales como mal comportamiento durante las comidas, 
Factores obtenidos a partir del método mínimos cuadrados no ponderados y promin. Los valores mayores a .30 se encuentran resaltados.

\begin{tabular}{rlcc}
\hline \multicolumn{1}{c}{ Ítems EADAN } & Factor 1 & Factor 2 \\
\hline 1: & ¿Cómo describe las comidas con su hijo? & .156 & $\mathbf{. 6 7 1}$ \\
6: & ¿Cómo se comporta su hijo en las comidas? & -.386 & $\mathbf{. 9 5 5}$ \\
10: & ¿Tiene que forzarlo a comer/beber? & .254 & $\mathbf{. 5 8 9}$ \\
13: & ¿Cómo afecta la alimentación su relación con su hijo? & .037 & $\mathbf{. 7 0 2}$ \\
9: ¿Tiene que entretenerlo o perseguirlo para que coma? & .242 & $\mathbf{. 5 5 5}$ \\
14: & ¿Qué efecto tiene la alimentación de su hijo en las relaciones familiares? & .064 & $\mathbf{. 6 1 0}$ \\
4: & ¿En qué momento de la comida su hijo se niega a comer? & $\mathbf{. 3 7 2}$ & $\mathbf{. 3 4 3}$ \\
5: & ¿Cuánto tiempo le lleva comer a su hijo? & -.257 & $\mathbf{. 4 1 9}$ \\
2: & ¿Está preocupada por la alimentación de su hijo? & $\mathbf{. 7 5 9}$ & -.079 \\
3: & ¿Cuánto apetito tiene su hijo? & $\mathbf{. 6 2 2}$ & .105 \\
11: & ¿Cómo mastica/succiona su hijo? & $\mathbf{. 7 2 6}$ & -.287 \\
12: & ¿Cómo describe el crecimiento de su hijo? & $\mathbf{. 5 3 6}$ & -.027 \\
7: & ¿Tiene arcadas, escupe o vomita con ciertos tipos de comidas? & $\mathbf{. 4 7 0}$ & -.002 \\
8: & ¿Guarda la comida en la boca sin tragársela? & $\mathbf{. 3 0 8}$ & .136 \\
\hline
\end{tabular}

influencia en las relaciones familiares, estrategias como forzar al niño a comer, entretenerlo o perseguirlo. Se puede observar que en el ítem 4 los valores para cada factor son cercanos, por lo que se infiere que este ítem es estadísticamente débil y no tendría un peso muy significativo en la constitución de los factores.

\section{Discusión}

En el presente artículo se exponen las características psicométricas de la EADAN, que consiste en la adaptación argentina y la validación en nuestra población de la MCH-FS. Los resultados expuestos sustentan la utilización de la EADAN como un instrumento para la detección de dificultades en la alimentación en niños. El coeficiente alfa de Cronbach fue satisfactorio para la EADAN, lo que indica una buena consistencia interna.

En nuestra investigación se obtuvieron dos factores que subyacen a las variables estudiadas. El Factor 1 se refiere a las causas y consecuencias de las dificultades en la alimentación y el segundo factor a las preocupaciones parentales y estrategias utilizadas frente a las dificultades en la alimentación. Se puede advertir que el ítem 4 sería estadísticamente débil ya que no presenta diferencias tan marcadas entre los valores para un factor y para otro, por lo que sería necesario revisar en estudios posteriores si es necesario retirarlo o modificarlo para ser incorporado en el instrumento.

Se pueden observar diferencias significativas tanto en los puntajes medios por ítem como en el puntaje medio total obtenido en el grupo de niños con dificultades en la alimentación y el grupo de niños sin dificultades. Esto evidencia que 
en la muestra argentina los valores del grupo con dificultades son claramente superiores a los valores del grupo sin dificultades, lo que coincide con los resultados de los estudios realizados en Canadá (Ramsay et al., 2011) y en Tailandia (Benjasuwantep et al., 2015).

Al comparar los puntajes medios de los ítems obtenidos por los grupos con y sin dificultades, se puede observar que ciertos comportamientos considerados problemáticos en torno a la alimentación se encuentran presentes en ambos grupos. Esto coincide con lo señalado por Ramsay et al. (2011) respecto de que la diferencia estaría en la frecuencia con la que se presentan estos comportamientos o en la percepción de los padres respecto de la severidad de los mismos y las reacciones ante ellos. En nuestra investigación, la frecuencia de las dificultades en la alimentación es del $12 \%$, inferior a lo que se estima en la literatura (Arvedson, como se citó en Barton et al., 2017) y a los resultados obtenidos en el estudio canadiense (Ramsay et al., 2011).

Al comparar los valores medios por ítem y el valor medio del puntaje total de la investigación argentina con los estudios anteriores se puede notar que, respecto de la muestra normativa de Canadá, los valores argentinos por ítem y el puntaje total son claramente inferiores. Esto podría indicar que las conductas problemáticas son menos frecuentes o menos intensas en nuestra muestra; sin embargo, esto requiere ser estudiado en mayor profundidad. No ocurre lo mismo con el estudio holandés y el tailandés, en los que el puntaje medio es inferior en algunos ítems y superior en otros. Esto podría estar relacionado con las diferencias en el rango de edad de los niños estudiados, ya que en Canadá, al igual que en el presente estudio, se evaluaron niños de entre los 6 meses y los 6 años 11 meses; en cambio, en las otras dos investigaciones el rango abarcó hasta los 4 años de edad.
En la presente investigación se encontró que los niños que fueron prematuros extremos obtuvieron los puntajes medios por ítem y el puntaje total más elevado dentro de la muestra en estudio. Esto corrobora lo señalado en la literatura (Hvelplund et al., 2016) respecto de la EG inferior a 28 semanas como un factor que puede incrementar el riesgo de aparición de dificultades en la alimentación. Por otro lado, es importante mencionar que en esta investigación no se hallaron diferencias significativas en cuanto a la frecuencia con la que se presentaron las dificultades en la alimentación entre niños prematuros y a término (12\% vs 13\%). Esto coincide con lo señalado por Van Dijk et al. (2016), quienes obtuvieron que la mayoría de los prematuros estudiados en su investigación no presentaban problemas de alimentación o bien presentaban problemas leves. Si bien estos resultados parecen contradecir las numerosas investigaciones que señalan a la prematurez como un factor de riesgo para este tipo de problemas, sería necesario considerar la posibilidad de ampliar la cantidad de prematuros evaluados para profundizar este análisis. Sin embargo, resulta evidente que a medida que disminuye la edad gestacional al nacer aumentan los puntajes medios en los ítems del cuestionario. Los resultados permiten señalar que, si bien el puntaje total del instrumento es relevante, resulta fundamental considerar los valores obtenidos en los ítems individuales, ya que esto permitirá delinear el tratamiento más oportuno en cada caso.

Es importante señalar que los resultados obtenidos en la presente investigación coinciden con los hallazgos de Van Dijk et al. (2011) respecto del incremento de la media del puntaje total a medida que aumenta la edad de los niños. Esto se puede notar en la muestra general, en la que la media va incrementándose a partir de los 6 meses para alcanzar su pico a los 4 años. De igual manera, en el grupo de 33 niños que tuvieron di- 
ficultades en la alimentación, el valor medio más elevado se obtuvo entre los 3 años y 1 mes y los 4 años, lo que coincide con lo obtenido por los autores holandeses. Esto podría estar relacionado con las características de la etapa por la que atraviesan los niños, caracterizada por un mayor oposicionismo y berrinches.

Una limitación del presente estudio es el tamaño de la muestra, que no permitiría realizar generalizaciones a la población respecto de la frecuencia de los problemas de alimentación en los niños. Otra limitación es que los cuestionarios fueron completados por los padres mientras esperaban con sus hijos para la consulta pediátrica $o$ una vez finalizada la misma. Esto supone que los padres tenían a sus hijos en brazos, en ocasiones los alimentaban o estaban pendientes de que no se alejaran, lo que podría afectar la atención y la información brindada en el cuestionario. Tal vez sería conveniente ofrecer a los padres que completen el cuestionario en el hogar y luego lo devuelvan o enviárselos por correo electrónico.

A partir de la presente investigación se puede concluir que la EADAN es un instrumento de cribado confiable, válido para la detección de dificultades en la alimentación en niños de entre 6 meses y 6 años y 11 meses de la población argentina. Se trata de un instrumento de gran utilidad para su implementación en la consulta pediátrica, tanto en los controles médicos de niños sanos como los de nacidos prematuros. Este instrumento resulta de gran importancia en nuestro país ya que permitiría cubrir la vacancia respecto de instrumentos adaptados y validados que permitan una rápida detección de las dificultades en la alimentación en los niños pequeños. Se trata de un instrumento de fácil administración y fácil comprensión por los padres en nuestra población.

En investigaciones futuras sería recomendable ampliar la muestra tanto en el número de sujetos como en las localidades en las que se ad- ministre el instrumento a fin de poder desarrollar baremos locales para los profesionales de la salud. La relevancia de continuar investigando en esta dirección surge del conocimiento respecto del impacto negativo que tienen las dificultades en la alimentación en el crecimiento y el desarrollo de los niños, así como en las relaciones familiares. La detección temprana de estas problemáticas contribuiría a mejorar la salud física de los niños, así como los vínculos familiares, ofreciendo una oportunidad para que todos los niños puedan acceder a un crecimiento y desarrollo saludable.

\section{Referencias}

Adamson, M., Morawska, A., \& Sanders, M. R. (2013). Childhood feeding difficulties: A randomized controlled trial of a group-based parenting intervention. Journal of Developmental \& Behavioral Pediatrics, 34(5), 293-302. doi: 10.1097/ DBP.0b013e3182961a38

Barton, C., Bickell, M., \& Fucile, S. (2017). Pediatric oral motor feeding assessments: A systematic review. Physical \& Occupational Therapy in Pediatrics, 38(2), 190-209. doi: 10.1080/01942638.2017.1290734

Benjasuwantep, B., Rattanamongkolgul, S., \& Ramsay, M. (2015). The Thai version of the Montreal Children's Hospital Feeding Scale (MCH-FS): Psychometric properties. Journal of Medical Association of Thailand, 98(2), 163-169. Recuperado de http://www.jmatonline.com/index.php/jmat

Blisseth, J. (2011). Relationships between parenting style, feeding style and feeding practices and fruit and vegetable consumption in early childhood. Appetite, 57(3), 826-831. doi: 10.1016/j.appet.2011.05.318

Fernández-Gallardo, M. A., Rojas-Contreras, D. P., \& Vargas-Keith, J. F. (2017). Development of feeding skills in preterm infants: A critical literature review. Revista CEFAC, 19(4), 539-550. doi: 10.1590/1982-021620171946417 
Goh, D. \& Jacob, A. (2012). Perception of picky eating among children in Singapore and its impact on caregivers: A questionnaire survey. Asia Pacific Family Medicine, 11(5), 1-8. doi: 10.1186/1447-056X-11-5

Horn, J. L. (1965). A rationale and test for the number of factors in factor analysis. Psychometrika, 30(2), 179185. doi: $10.1007 /$ bf02289447

Hvelplund, C., Hansen, B. M., Vinkel-Koch, S., Andersson, M., \& Skovgaard, A. M. (2016). Perinatal risk factors for feeding and eating disorders in children aged 0 to 3 years. Pediatrics, 137(2). doi: 10.1542/ peds.2015-2575

IBM Corp. (2012). IBM SPSS Statistics for Windows, Version 21.0. [software de cómputo]. Armonk, NY: IBM Corp.

Johnson, R., \& Harris, G. (2004). A preliminary study of the predictors of feeding problems in late infancy. Journal of Reproductive and Infant Psychology, 22(3), 183-188. doi: 10.1080/02646830410001723760

Lindberg, L., Bohlin, G., \& Hagekull, B. (1991). Early feeding problems in a normal population. International Journal of Eating Disorders, 10(4), 395-405. doi: 10.1002/1098-108x(199107)10:4<395::aideat2260100404>3.0.co;2-a

Lorenzo-Seva, U. (1999). Promin: A method for oblique factor rotation. Multivariate Behavioral Research, 34(3), 347-356. doi: 10.1207/S15327906MBR3403_3

Lorenzo-Seva, U., \& Ferrando, P. J. (2013). FACTOR 9.2: A comprehensive program for fitting exploratory and semiconfirmatory factor analysis and IRT models. Applied Psychological Measurement, 37(6), 497498. doi: 10.1177/0146621613487794

McDermott, B. M., Mamun, A. A., Najman, J. M., Williams, G. M., O’Callaghan, M. J., \& Bor, W. (2008). Preschool children perceived by mothers as irregular eaters: Physical and psychosocial predictors from a birth cohort study. Journal of Developmental and Behavioral Pediatrics, 29(3), 197-205. doi: 10.1097/ DBP.0b013e318163c388

Ramsay, M. (2013). Feeding skill, appetite and feeding behaviours of infants and young children and their im- pact on growth and psychosocial development. En R. E. Tremblay, R. G. Barr \& R. DeV. Peters (Eds.), Encyclopedia on Early Childhood Development [online] (1-8). Montreal, Quebec: Centre of Excellence for Early Childhood Development. Recuperado de http://www.child-encyclopedia.com

Ramsay, M., Martel, C., Porporino, M., \& Zygmuntowicz, C. (2011). The Montreal Children's Hospital Feeding Scale: A brief bilingual screening tool for identifying feeding problems. Paediatrics and Child Health, 16(3), 147-e17. doi: 10.1093/pch/16.3.147

Rommel, N., De Meyer, A. M., Feenstra, L., \& Veereman-Wauters, G. (2003). The complexity of feeding problems in 700 infants and young children presenting to a tertiary care institution. Journal of Pediatric Gastroenterology and Nutrition, 37(1), 75-84. doi: 10.1097/00005176-200307000-00014

Sanders, M. R., Patel, R. K., Le Grice, B., \& Shepherd, R. W. (1993). Children with persistent feeding difficulties: An observational analysis of the feeding interactions of problem and non-problem eaters. Health Psychology, 12(1), 64-73. doi: 10.1037//0278-6133.12.1.64

Saxton, J., Carnell, S., van Jaarsveld, C. H. M., \& Wardle, J. (2009). Maternal education is associated with feeding style. Journal of the American Dietetic Association, 109(5), 894-898. doi: 10.1016/j.jada.2009.02.010

Van Dijk, M., Bruinsma, E., \& Hauser, M. P. (2016). The relation between child feeding problems as measured by parental report and mealtime behavior observation: A pilot study. Appetite, 99, 262-267. doi: 10.1016/j.appet.2016.01.026

Van Dijk, M., Timmerman, M. E., Martel, C., \& Ramsay, M. (2011). Towards the development of a Dutch screening instrument for the detection of feeding problems in young children. Netherlands Journal of Psychology, 66(4), 112-119. Recuperado de https://www.rug.nl/ research/portal/journals/netherlands-journal-of-psychology(11473cbc-a542-47fc-acbb-736cd32ce42c)/ publications.html 